\title{
DEFICIENCIES OF THE ASSOCIATED CURVES OF A HOLOMORPHIC CURVE IN THE PROJECTIVE SPACE
}

\author{
KIYOSHI NIINO
}

\begin{abstract}
Let ${ }_{k} x$ be the nonconstant associated holomorphic curve of rank $k(1 \leqq k \leqq n-1)$ of a transcendental holomorphic curve $x$ : $\mathbf{C}$ $\rightarrow P_{n} \mathbf{C}$. It is proved that if $1 \leqq k \leqq n-2$ and $A_{j}^{k} \in P_{l(k)-1} \mathbf{C}, j=1, \ldots$, $2 l(k)-2\left(l(k)=\left(\begin{array}{c}n+1 \\ k+1\end{array}\right)\right)$ are in general position and $\left\langle k x, A_{j}^{k}\right\rangle \neq \equiv 0$ for all $A_{j}^{k}$, then $\sum_{j=1}^{2 l(k)-2} \delta_{k}\left(A_{j}^{k}\right) \leqq 2 l(k)-3$ and that in the case when $k=n-1$, $\sum_{A^{n-1}} \delta_{n-1}\left(A^{n-1}\right) \leqq l(n-1)$, where $\left\{A^{n-1}\right\}$ is a finite subset of $P_{l(n-1)-1} \mathrm{C}$ in general position such that $\left\langle_{n-1} x, A^{n-1}\right\rangle \not \equiv 0$ for all $A^{n-1}$. These are sharp.
\end{abstract}

1. The theory of holomorphic (or meromorphic) curves was initiated by $\mathrm{H}$. and J. Weyl in 1938 [7], [8]. Its main problem, the proof of the so-called defect relations, was solved by Ahlfors [1]. A modern detailed treatment was given by $\mathrm{Wu}[9]$. It is a natural generalization of the Picard-Borel-Nevanlinna theory. On the other hand, a generalization of the Nevanlinna theory to systems of entire functions had been tried by Cartan in 1933 [2].

If a system of entire functions $\left(x_{0}, x_{1}, \ldots, x_{n}\right)$ is given, then we can see it as a reduced representation of a holomorphic curve $x: \mathbf{C} \rightarrow P_{n} \mathbf{C}$. By the reasoning in [2, pp. 7-8] and [8, pp. 81-84] or [9, pp. 104-105], the characteristic function defined by Cartan for the system of entire functions is essentially equal to the order function defined by Weyl for the corresponding holomorphic curve in the projective space. Hence the results on systems of entire functions can be restated by the statements on holomorphic curves in the projective space.

The defect relation for nondegenerate holomorphic curves in the projective space was obtained by Ahlfors [1] and Cartan [2]. But it seems that a defect relation for degenerate holomorphic curves in the projective space has not been yet obtained in a sharp form. Concerning it there are a result of Toda [5] and a conjecture of Cartan [2].

Recently, Toda [6] made another contribution to the study of degenerate holomorphic curves which is in turn a generalization of the result of the author and Ozawa [3], [4] on entire algebroid functions. In the language of holomorphic curves (cf. [9]), Toda's result concerns the defects of the original curve.

Received by the editors June 2, 1975 and, in revised form, December 22, 1975.

AMS (MOS) subject classifications (1970). Primary 30A70.

Key words and phrases. Holomorphic curve, associated curve of rank $k$, projective space, order function, defect (deficiency), general position, degenerate curve.

○ American Mathematical Society 1976 
This paper makes a similar study for the defects of the associated curves.

A result of Toda [6] is restated by the following statement on holomorphic curves in the projective space.

Theorem A. Let $x: \mathrm{C} \rightarrow P_{n} \mathbf{C}$ be a transcendental holomorphic curve and $\tilde{x}=\left(x_{0}, x_{1}, \ldots, x_{n}\right): \mathbf{C} \rightarrow \mathbf{C}^{n+1}-\{0\}$ its reduced representation. If there are $a_{j} \in P_{n} \mathbf{C}, j=1,2, \ldots, 2 n$, in general position such that $\left\langle x, a_{j}\right\rangle \not \equiv 0$ for all $j$ and $\sum_{j=1}^{2 n} \delta_{0}\left(a_{j}\right)>2 n-1$, then $\lambda=n-1$, where $\lambda$ is the maximum number of independent, linear relations with constant coefficients among the entire functions $x_{0}, x_{1}, \ldots, x_{n}$, and the entire functions $\left\langle x(z), a_{j}\right\rangle$ are divided into two classes having the following properties:

(i) either class contains $n$ entire functions,

(ii) the entire functions of the same class are proportional.

(For terminology and notations of the theory of holomorphic curves, see Wu's note [9].)

As corollary of Theorem A, we have

Theorem B. Let $x: \mathbf{C} \rightarrow P_{n} \mathbf{C}$ be a transcendental holomorphic curve. If $a_{j} \in P_{n} \mathbf{C}, j=1,2, \ldots, 2 n+1$, are in general position and $\left\langle x, a_{j}\right\rangle \not \equiv 0$ for all $j$, then we have $\sum_{j=1}^{2 n+1} \delta_{0}\left(a_{j}\right) \leqq 2 n$.

Theorem $\mathrm{B}$ is sharp in the sense that there are a holomorphic curve $x: \mathbf{C} \rightarrow P_{n} \mathbf{C}$ and $a_{j} \in P_{n} \mathbf{C}, j=1, \ldots, 2 n$, in general position such that $\left\langle x, a_{j}\right\rangle \not \equiv 0$ for all $j$ and

$$
\sum_{j=1}^{2 n} \delta_{0}\left(a_{j}\right)=2 n .
$$

In fact, put $x_{0}(z)=e^{z}-1, x_{1}(z) \equiv \cdots \equiv x_{n-1}(z) \equiv 0, x_{n}(z)=-e^{z}+2$ and $\tilde{a}_{j}=\left(\alpha_{j}^{n}, \alpha_{j}^{n-1}, \ldots, \alpha_{j}, 1\right)$, where $\eta=\exp (2 \pi i / n)$ and $\alpha_{j}=\eta^{j-1}$ for $j=1$, $2, \ldots, n$ and $\alpha_{j}=2^{1 / n} \eta^{j-n-1}$ for $j=n+1, \ldots, 2 n$. Then $\tilde{x}=\left(x_{0}, x_{1}, \ldots\right.$, $\left.x_{n}\right)$ satisfies $\left\langle\tilde{x}(z), \tilde{a}_{j}\right\rangle=1$ for $j=1, \ldots, n$ and $\left\langle\tilde{x}(z), \tilde{a}_{j}\right\rangle=e^{z}$ for $j=n+1$, $\ldots, 2 n$. It is clear that $\pi\left(\tilde{a}_{j}\right)=a_{j} \in P_{n} \mathbf{C}, j=1, \ldots, 2 n$, are in general position and the holomorphic curve $x: \mathbf{C} \rightarrow P_{n} \mathbf{C}$ induced by $\tilde{x}$ satisfies $\left\langle x(z), a_{j}\right\rangle \neq 0$ for all $z \in \mathbf{C}$ and $j=1, \ldots, 2 n$. Hence we have $\delta_{0}\left(a_{j}\right)=1$ for $j=1, \ldots, 2 n$ and so (1.1). Thus we obtain a desired example.

In this paper we shall prove

TheOREM 1. Let ${ }_{k} x: \mathbf{C} \rightarrow G(n, k) \subseteq P_{l(k)-1} \mathbf{C}\left(l(k)=\left(\begin{array}{c}n+1 \\ k+1\end{array}\right)\right)$ be the nonconstant associated holomorphic curve of rank $k(1 \leqq k \leqq n-2)$ of a transcendental holomorphic curve $x: \mathbf{C} \rightarrow P_{n} \mathbf{C}$. If $A_{j}^{k} \in P_{l(k)-1} \mathbf{C}, j=1,2, \ldots, 2 l(k)-2$, are in general position and $\left\langle_{k} x, A_{j}^{k}\right\rangle \neq \equiv$ for all $j$, then we have

$$
\sum_{j=1}^{2 l(k)-2} \delta_{k}\left(A_{j}^{k}\right) \leqq 2 l(k)-3 .
$$

Theorem 1 is sharp in the following sense: 
Theorem 2. There are a holomorphic curve $x: \mathbf{C} \rightarrow P_{n} \mathbf{C}$ and $A_{j}^{k} \in P_{l(k)-1} \mathbf{C}, j$ $=1,2, \ldots, 2 l(k)-3$, in general position such that $\left\langle_{k} x, A_{j}^{k}\right\rangle \not \equiv 0$ for all $j$ and

$$
\sum_{j=1}^{2 l(k)-3} \delta_{k}\left(A_{j}^{k}\right)=2 l(k)-3
$$

for some $n$ and $k$.

Theorem 1 is not true in the case $k=0$. Because $2 l(k)-2=2 n$ for $k=0$ and we have already had a holomorphic curve $x: \mathbf{C} \rightarrow P_{n} \mathbf{C}$ satisfying (1.1).

For the case $k=n-1$, we shall prove

Theorem 3. Let ${ }_{n-1} x: \mathrm{C} \rightarrow G(n, n-1) \subseteq P_{l(n-1)-1} \mathrm{C}$ be the nonconstant associated holomorphic curve of rank $n-1$ of a transcendental holomorphic curve $x: \mathbf{C} \rightarrow P_{n} \mathbf{C}$. If $\left\{A^{n-1}\right\}$ is a finite subset of $P_{l(n-1)-1} \mathbf{C}$ in general position and $\left\langle{ }_{n-1} x, A^{n-1}\right\rangle \not \equiv 0$ for all $A^{n-1}$, then

$$
\sum_{A^{n-1}} \delta_{n-1}\left(A^{n-1}\right) \leqq l(n-1)
$$

Theorem 3 is sharp in the following sense:

THEOREM 4. There are a holomorphic curve $x: \mathrm{C} \rightarrow P_{n} \mathbf{C}$ and $A_{j}^{n-1}$ $\in P_{l(n-1)-1} \mathbf{C}, j=1,2, \ldots, l(n-1)$, in general position such that $\left\langle_{n-1} x, A_{j}^{n-1}\right\rangle$ $\not \equiv 0$ for all $j$ and

$$
\sum_{j=1}^{l(n-1)} \delta_{n-1}\left(A_{j}^{n-1}\right)=l(n-1)
$$

for some $n$.

Remarks. Chen-Han Sungl has recently proved the conjecture of Cartan [2], that is,

THEOREM C. Let $x: \mathbf{C} \rightarrow P_{n} \mathbf{C}$ be a transcendental holomorphic curve, $\tilde{x}$ $=\left(x_{0}, x_{1}, \ldots, x_{n}\right): \mathbf{C} \rightarrow \mathbf{C}^{n+1}-\{0\}$ its reduced representation and $\lambda$ the maximum number of independent, linear relations with constant coefficients among entire functions $x_{0}, x_{1}, \ldots, x_{n}$. If $\{a\}$ is a finite subset of $P_{n} \mathbf{C}$ in general position and $\langle x, a\rangle \not \equiv 0$ for all $a$, then

$$
\sum_{a} \delta_{0}(a) \leqq n+1+\lambda
$$

We can deduce from Theorem $\mathrm{C}$, the proof of our Theorem 1 given in $\$ 2$ and our Theorem 2 that

THEOREM 1'. Let ${ }_{k} x: \mathbf{C} \rightarrow G(n, k) \subseteq P_{l(k)-1} \mathbf{C}$ be the nonconstant associated holomorphic curve of rank $k(1 \leqq k \leqq n-2)$ of a transcendental holomorphic curve $x: \mathbf{C} \rightarrow P_{n} \mathbf{C}$. If $\left\{A^{k}\right\}$ is a finite subset of $P_{l(k)-1} \mathbf{C}$ in general position and $\left\langle{ }_{k} x, A^{k}\right\rangle \not \equiv 0$ for all $A^{k}$, then

\footnotetext{
${ }^{1}$ Communication with the referee.
} 


$$
\sum_{A^{k}} \delta_{k}\left(A^{k}\right) \leqq 2 l(k)-3 .
$$

This is sharp.

The author wishes to express his heartiest thanks to the referee for his valuable advice and pointing out the result of Sung.

2. Proof of Theorem 1. Let $\tilde{x}=\left(x_{0}, x_{1}, \ldots, x_{n}\right)$ be a reduced representation of $x$. We denote by $\lambda$ the maximum number of independent, linear relations with constant coefficients among $x_{0}, \ldots, x_{n}$. Then there are $n+1-\lambda$ linearly independent functions in $\left\{x_{j}\right\}$, say $x_{0}, x_{1}, \ldots, x_{n-\lambda}$, such that the other functions are written by their linear combinations. Hence every ordered component of $X^{k}=\tilde{x} \wedge \tilde{x}^{(1)} \wedge \cdots \wedge \tilde{x}^{(k)}$ is written by a linear combination of $\left(\begin{array}{c}n+1-\lambda \\ k+1\end{array}\right)$ ordered components

$$
X^{k}\left(i_{0} i_{1} \cdots i_{k}\right)=\left\|x_{i_{0}} x_{i_{1}} \cdots x_{i_{k}}\right\|, \quad 0 \leqq i_{0}<i_{1}<\cdots<i_{k} \leqq n-\lambda,
$$

of $X^{k}$, where $\left\|x_{i_{0}} \cdots x_{i_{k}}\right\|$ is the Wronskian of $x_{i_{0}}, \ldots, x_{i_{k}}$. Since ${ }_{k} x: \mathbf{C}$ $\rightarrow G(n, k) \subseteq P_{l(k)-1} \mathbf{C}$ is not constant, we have $\left(\begin{array}{c}n+1-\lambda \\ k+1\end{array}\right) \geqq 2$ and, consequently,

$$
0 \leqq \lambda \leqq n-k-1
$$

Now suppose, to the contrary, that $\sum_{j=1}^{2 l(k)-2} \delta_{k}\left(A_{j}^{k}\right)>2 l(k)-3$. Then we apply Theorem A to the holomorphic curve ${ }_{k} x: \mathbf{C} \rightarrow P_{l(k)-1} \mathbf{C}$. Theorem A implies $\lambda_{k}=l(k)-2$, where $\lambda_{k}$ is the maximum number of independent, linear relations with constant coefficients among $l(k)$ ordered components $\left\{X^{k}\left(i_{0} \cdots i_{k}\right)\right\}$ of $X^{k}$. Hence there are two linearly independent components of $X^{k}$ in $\left\{X^{k}\left(i_{0} \cdots i_{k}\right)\right\}, 0 \leqq i_{0}<\cdots<i_{k} \leqq n-\lambda$, say $X^{k}(0 \cdots k)$ and $X^{k}\left(j_{0} \cdots j_{k}\right)$, such that the other components are written by their linear combinations. (2.1) and $k \geqq 1$ imply $\left(\begin{array}{c}n+1-\lambda \\ k+1\end{array}\right) \geqq 3$.

Assume that $\left(\begin{array}{c}n+1-\lambda \\ k+1\end{array}\right)=3$. Then we have $n+1-\lambda=k+2=3$ and so $n-\lambda=2$ and $k=1$. Since every ordered component of $X^{1}$ is written by a linear combination of $X^{1}\left(\begin{array}{ll}0 & 1)\end{array}\right)\left\|x_{0} x_{1}\right\|$ and $X^{1}\left(j_{0} j_{1}\right)=\left\|x_{j_{0}} x_{j_{1}}\right\|, 0 \leqq j_{0}$ $<j_{1} \leqq n-\lambda=2$, we have

$$
a\left\|x_{0} x_{1}\right\|+b\left\|x_{0} x_{2}\right\|+c\left\|x_{1} x_{2}\right\|=0
$$

and so $\left\{\left\|b x_{0}+c x_{1} a x_{1}+b x_{2}\right\|\right\} / b=0$ with three suitable constants $a, b$ and $c$ with $(a, b, c) \neq(0,0,0)$, say $b \neq 0$. Hence $b x_{0}+c x_{1}$ and $a x_{1}+b x_{2}$, that is, $x_{0}, x_{1}$ and $x_{2}$ are linearly dependent, which contradicts our assumption that $x_{0}, x_{1}$ and $x_{2}(n-\lambda=2)$ are linearly independent.

Next assume that $\left(\begin{array}{c}n+1-\lambda \\ k+1\end{array}\right) \geqq 4$. Then since $3 \leqq k+1 \leqq n-\lambda$, we have

$$
\begin{aligned}
\left\|x_{0} \cdots x_{k-2} x_{k-1} x_{k+1}\right\| & =a\left\|x_{0} \cdots x_{k}\right\|+b\left\|x_{j_{0}} \cdots x_{j_{k}}\right\|, \\
\left\|x_{0} \cdots x_{k-2} x_{k} x_{k+1}\right\| & =c\left\|x_{0} \cdots x_{k}\right\|+d\left\|x_{j_{0}} \cdots x_{j_{k}}\right\|
\end{aligned}
$$


with suitable constants $a, b, c$ and $d$. If $a d-b c=0$, then we have

$$
\left\|x_{0} \cdots x_{k-2} x_{k-1} x_{k+1}\right\|+\alpha\left\|x_{0} \cdots x_{k-2} x_{k} x_{k+1}\right\|=0
$$

and so

$$
\left\|x_{0} \cdots x_{k-2} x_{k-1}+\alpha x_{k} x_{k+1}\right\|=0
$$

with a suitable constant $\alpha$. Hence $x_{0}, \ldots, x_{k-2}, x_{k-1}+\alpha x_{k}$ and $x_{k+1}$, that is, $x_{0}, \ldots, x_{k+1}$ are linearly dependent, which contradicts our assumption that $x_{0}, \ldots, x_{n-\lambda}(k+1 \leqq n-\lambda)$ are linearly independent. If $a d-b c \neq 0$, then we have

$$
\alpha\left\|x_{0} \cdots x_{k}\right\|-d\left\|x_{0} \cdots x_{k-2} x_{k-1} x_{k+1}\right\|-b\left\|x_{0} \cdots x_{k-2} x_{k} x_{k+1}\right\|=0
$$

and so

$$
\left\|x_{0} \cdots x_{k-2} d x_{k-1}+b x_{k} \alpha x_{k}-d x_{k+1}\right\|=0,
$$

where $\quad \alpha=a d-b c \neq 0$. Hence $\quad x_{0}, \ldots, x_{k-2}, d x_{k-1}+b x_{k}$ and $\alpha x_{k}$ $-d x_{k+1}$, that is, $x_{0}, \ldots, x_{k+1}$ are linearly dependent, which is a contradiction.

Therefore we obtain

$$
\sum_{j=1}^{2 l(k)-2} \delta_{k}\left(A_{j}^{k}\right) \leqq 2 l(k)-3,
$$

which gives our Theorem 1.

3. Proof of Theorem 2. For $n=3$ and $k=1$ we shall give a holomorphic curve $x: \mathbf{C} \rightarrow P_{n} \mathbf{C}$ and $A_{j}^{k} \in P_{l(k)-1} \mathbf{C}, j=1, \ldots, 2 l(k)-3$, in general position satisfying (1.2). We have $l(k)=6$ and $2 l(k)-3=9$ for $n=3$ and $k$ $=1$.

Put

$$
\begin{array}{ll}
x_{0}(z)=15^{1 / 2} i z e^{z / 2}, & x_{1}(z)=15^{1 / 2} i e^{z / 2}, \\
x_{2}(z)=-(1 / 15)^{1 / 2} i\left(e^{z}-1\right) e^{z / 2}, & x_{3}(z)=16(1 / 15)^{1 / 2} i\left(e^{z}-1\right) e^{z / 2} .
\end{array}
$$

Let $x: \mathbf{C} \rightarrow P_{3} \mathbf{C}$ be the holomorphic curve induced by $\tilde{x}=\left(x_{0}, x_{1}, x_{2}, x_{3}\right)$. Then $X^{1}=\tilde{x} \wedge \tilde{x}^{(1)}$ has the following ordered components:

$$
\begin{aligned}
& X^{1}\left(\begin{array}{ll}
0 & 2
\end{array}\right)=\left\|x_{0} x_{2}\right\|=x_{0} x_{2}^{\prime}-x_{0}^{\prime} x_{2}=e^{z}+(z-1) e^{2 z}, \quad X^{1}\left(\begin{array}{ll}
1 & 2
\end{array}\right)=e^{2 z}, \\
& X^{1}\left(\begin{array}{ll}
0 & 1)
\end{array}\right)=15 e^{z}, \quad X^{1}\left(\begin{array}{ll}
0 & 3
\end{array}\right)=-16\left(e^{z}+(z-1) e^{2 z}\right), \quad X^{1}\left(\begin{array}{ll}
2 & 3
\end{array}\right)=0, \\
& X^{1}\left(\begin{array}{ll}
1 & 3
\end{array}\right)=-16 e^{2 z} .
\end{aligned}
$$

Considering $X^{1}$ as a point of $\mathbf{C}^{6}$, we put $X^{1}=\left(X^{1}\left(\begin{array}{ll}0 & 2\end{array}\right), X^{1}\left(\begin{array}{ll}1 & 2\end{array}\right), X^{1}\left(\begin{array}{ll}0 & 1\end{array}\right)\right.$, $\left.X^{1}\left(\begin{array}{ll}2 & 3\end{array}\right), X^{1}\left(\begin{array}{ll}0 & 3\end{array}\right), X^{1}\left(\begin{array}{ll}1 & 3\end{array}\right)\right) \in \mathbf{C}^{6}$. Further put $\tilde{A}_{j}^{1}=\left(\bar{w}_{j}^{5}, \bar{w}_{j}^{4}, \ldots, \bar{w}_{j}, 1\right) \in \mathbf{C}^{6}$. Then we have 


$$
\begin{aligned}
\left\langle X^{1}, \tilde{A}_{j}^{1}\right\rangle= & \left(e^{z}+(z-1) e^{2 z}\right) w_{j}^{5}+e^{2 z} w_{j}^{4}+15 e^{z} w_{j}^{3} \\
& -16\left(e^{z}+(z-1) e^{2 z}\right) w_{j}-16 e^{2 z} \\
= & w_{j}\left(w_{j}^{2}-1\right)\left(w_{j}^{2}+16\right) e^{z}+\left(w_{j}^{4}-16\right)\left((z-1) w_{j}+1\right) e^{2 z} .
\end{aligned}
$$

Put $\quad w_{1}=0, w_{2}=1, w_{3}=-1, w_{4}=4 i, w_{5}=-4 i, w_{6}=2, w_{7}=-2, w_{8}$ $=2 i$ and $w_{9}=-2 i$. Then it is clear that $A_{j}^{1}={ }_{1} \pi\left(\tilde{A}_{j}^{1}\right) \in P_{5} \mathbf{C}, j=1, \ldots, 9$, are in general position. And we have

$$
\begin{aligned}
& \left\langle X^{1}, \tilde{A}_{1}^{1}\right\rangle=-16 e^{2 z},\left\langle X^{1}, \tilde{A}_{2}^{1}\right\rangle=-15 z e^{2 z},\left\langle X^{1}, \tilde{A}_{3}^{1}\right\rangle=15(z-2) e^{2 z}, \\
& \left\langle X^{1}, \tilde{A}_{4}^{1}\right\rangle=240(4 i z+1-4 i) e^{2 z},\left\langle X^{1}, \tilde{A}_{5}^{1}\right\rangle=-240(4 i z-1-4 i) e^{2 z}, \\
& \left\langle X^{1}, \tilde{A}_{6}^{1}\right\rangle=120 e^{z},\left\langle X^{1}, \tilde{A}_{7}^{1}\right\rangle=-120 e^{z},\left\langle X^{1}, \tilde{A}_{8}^{1}\right\rangle=-120 i e^{z}, \\
& \left\langle X^{1}, \tilde{A}_{9}^{1}\right\rangle=120 i e^{z} .
\end{aligned}
$$

Thus $\left\langle X^{1}, \tilde{A}_{j}^{1}\right\rangle$ has no zero or only one zero. Hence $\delta_{1}\left(A_{j}^{1}\right)=1$ for $j=1, \ldots$, 9, which gives (1.2) for $n=3$ and $k=1$.

Thus the proof of Theorem 2 is complete.

4. Proof of Theorem 3. Suppose, to the contrary, that $\sum_{A^{n-1}} \delta_{n-1}\left(A^{n-1}\right)$ $>l(n-1)$. Then we apply Theorem 5.13 (defect relations) in [9] to the holomorphic curve ${ }_{n-1} x: \mathbf{C} \rightarrow P_{l(n-1)-1} \mathbf{C}$. It follows that the holomorphic curve ${ }_{n-1} x$ must be degenerate, that is, there is a linear relation among the ordered components of $X^{n-1}=\tilde{x} \wedge \cdots \wedge \tilde{x}^{(n-1)}$, where $\tilde{x}=\left(x_{0}, \ldots, x_{n}\right)$ is a reduced representation of $x$. Hence we have

$$
\sum_{j=0}^{n} \alpha_{j} X^{n-1}(0 \cdots \hat{j} \cdots n)=\sum_{j=0}^{n} \alpha_{j}\left\|x_{0} \cdots \hat{x}_{j} \cdots x_{n}\right\|=0
$$

where - means "omit". We denote by $h+1(0 \leqq h \leqq n)$ the number of nonzero $\alpha_{j}$. Then we may assume, without loss of generality, that $\alpha_{0} \alpha_{1} \cdots \alpha_{h}$ $\neq 0$. Then from (4.1) we have

$$
\sum_{j=0}^{h} \alpha_{j}\left\|x_{0} \cdots \hat{x}_{j} \cdots x_{n}\right\|=0
$$

and consequently

$$
\frac{\left\|\alpha_{1} x_{0}+\alpha_{0} x_{1} \alpha_{2} x_{1}+\alpha_{1} x_{2} \cdots \alpha_{h} x_{h-1}+\alpha_{h-1} x_{h} x_{h+1} \cdots x_{n}\right\|}{\left(\alpha_{1} \cdots \alpha_{h-1}\right)}=0 .
$$

Therefore $\alpha_{1} x_{0}+\alpha_{0} x_{1}, \ldots, \alpha_{h} x_{h-1}+\alpha_{h-1} x_{h}, x_{h+1}, \ldots, x_{n}$, that is, $x_{0}, \ldots$, $x_{n}$ are linearly dependent. Then we deduce that the ordered components of $X^{n-1}$ are proportional. Hence ${ }_{n-1} x: \mathbf{C} \rightarrow G(n, n-1) \subseteq P_{l(n-1)-1} \mathbf{C}$ is a constant map, which contradicts our assumption. Therefore we have 


$$
\sum_{A^{n-1}} \delta_{n-1}\left(A^{n-1}\right) \leqq l(n-1),
$$

which gives our Theorem 3 .

5. Proof of Theorem 4. For $n=3$ we shall give a holomorphic curve $x: \mathbf{C} \rightarrow P_{n} \mathbf{C}$ and $A_{j}^{n-1} \in P_{l(n-1)-1} \mathbf{C}, j=1, \ldots, l(n-1)$, in general position satisfying $\left\langle_{n-1} x, A_{j}^{n-1}\right\rangle \not \equiv 0$ and (1.3). We have $l(n-1)=4$ for $n=3$.

Put

$$
\begin{array}{ll}
x_{0}(z)=\left(-3 e^{2 z}-9 e^{z}+3+e^{-z}\right) / 18^{2 / 3}, & x_{1}(z)=\left(9 e^{z}+3-2 e^{-z}\right) / 18^{2 / 3}, \\
x_{2}(z)=\left(-9 e^{z}+3+4 e^{-z}\right) / 18^{2 / 3}, & x_{3}(z)=\left(9 e^{z}+3-8 e^{-z}\right) / 18^{2 / 3} .
\end{array}
$$

Let $x: \mathbf{C} \rightarrow P_{3} \mathbf{C}$ be the holomorphic curve induced by $\tilde{x}=\left(x_{0}, x_{1}, x_{2}, x_{3}\right)$. Then $X^{2}=\tilde{x} \wedge \tilde{x}^{(1)} \wedge \tilde{x}^{(2)}$ has the following ordered components:

$$
\begin{aligned}
& X^{2}\left(\begin{array}{lll}
0 & 1 & 2
\end{array}\right)=\left\|x_{0} x_{1} x_{2}\right\| \\
&=x_{0} x_{1}^{\prime} x_{2}^{\prime \prime}+x_{1} x_{2}^{\prime} x_{0}^{\prime \prime}+x_{2} x_{0}^{\prime} x_{1}^{\prime \prime}-x_{0}^{\prime \prime} x_{1}^{\prime} x_{2}-x_{1}^{\prime \prime} x_{2}^{\prime} x_{0}-x_{2}^{\prime \prime} x_{0}^{\prime} x_{1} \\
&=e^{3 z}+e^{2 z}+e^{z}+1, \\
& X^{2}\left(\begin{array}{lll}
0 & 1 & 3
\end{array}\right)=-3 e^{2 z}-e^{z}-2, \quad X^{2}\left(\begin{array}{lll}
0 & 2 & 3
\end{array}\right)=-e^{3 z}+2 e^{2 z}-2 e^{z}-1, \\
& X^{2}\left(\begin{array}{lll}
1 & 2 & 3
\end{array}\right)=2 .
\end{aligned}
$$

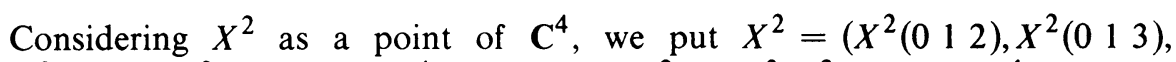
$\left.X^{2}\left(\begin{array}{lll}0 & 2 & 3\end{array}\right), X^{2}\left(\begin{array}{lll}1 & 2 & 3\end{array}\right)\right) \in \mathbf{C}^{4}$. Further put $\tilde{A}_{j}^{2}=\left(\bar{w}_{j}^{3}, \bar{w}_{j}^{2}, \bar{w}_{j}, 1\right) \in \mathbf{C}^{4}$. Then we have

$$
\begin{aligned}
\left\langle X^{2}, \tilde{A}_{j}^{2}\right\rangle= & \left(e^{3 z}+e^{2 z}+e^{z}+1\right) w_{j}^{3}+\left(-3 e^{2 z}-e^{z}-2\right) w_{j}^{2} \\
& +\left(-e^{3 z}+2 e^{2 z}-2 e^{z}-1\right) w_{j}+2 \\
= & w_{j}\left(w_{j}-1\right)\left(w_{j}+1\right) e^{3 z}+w_{j}\left(w_{j}-1\right)\left(w_{j}-2\right) e^{2 z} \\
& +w_{j}\left(w_{j}+1\right)\left(w_{j}-2\right) e^{z}+\left(w_{j}-1\right)\left(w_{j}+1\right)\left(w_{j}-2\right) .
\end{aligned}
$$

Put $w_{1}=0, w_{2}=1, w_{3}=-1$ and $w_{4}=2$. Then it is clear that $A_{j}^{2}={ }_{2} \pi\left(\tilde{A}_{j}^{2}\right)$ $\in P_{3} \mathbf{C}, j=1, \ldots, 4$, are in general position. And we have

$$
\begin{aligned}
& \left\langle X^{2}, \tilde{A}_{1}^{2}\right\rangle=2, \quad\left\langle X^{2}, \tilde{A}_{2}^{2}\right\rangle=-2 e^{z}, \\
& \left\langle X^{2}, \tilde{A}_{3}^{2}\right\rangle=-6 e^{2 z},\left\langle X^{2}, \tilde{A}_{4}^{2}\right\rangle=6 e^{3 z} .
\end{aligned}
$$

Thus $\left\langle X^{2}, \tilde{A}_{j}^{2}\right\rangle$ has nd zero. Hence $\delta_{2}\left(A_{j}^{2}\right)=1$ for $j=1, \ldots, 4$, which gives (1.3) for $n=3$. Thus the proof of Theorem 4 is complete. 


\section{REFERENCES}

1. L. V. Ahlfors, The theory of meromorphic curves, Acta Soc. Sci. Fenn. Ser. A 3 (1941), no. 4, 31 pp. MR 2, 357.

2. H. Cartan, Sur les zeros des combinaisons linéaires de p fonctions holomorphes données, Mathematica 7 (1933), 5-31.

3. K. Niino and M. Ozawa, Deficiencies of an entire algebroid function. I, Kōdai Math. Sem. Rep. 22 (1970), 98-113. MR 43 \#2226.

4. - Deficiencies of an entire algebroid function. II, Kōdai Math. Sem. Rep. 22 (1970), 178-187. MR 43 \#2226.

5. N. Toda, Sur les combinaisons exceptionnelles de fonctions holomorphes; applications aux fonctions algébroides, Tôhoku Math. J. (2) 22 (1970), 290-319. MR 42 \#6238.

6. - Sur quelques combinaisons linéaires exceptionnelles au sens de Nevanlinna. II, J. Math. Soc. Japan 25 (1973), 158-167. MR 47 \#3680.

7. H. Weyl and J. Weyl, Meromorphic curves, Ann. of Math. (2) 39 (1938), 516-538.

8. - Meromorphic functions and analytic curves, Ann. of Math. Studies, no. 12, Princeton, Univ. Press, Princeton, N. J., 1970.

9. H. Wu, The equidistribution theory of holomorphic curves, Ann. of Math. Studies, no. 64, Princeton Univ. Press, Princeton, N. J.; Univ. of Tokyo Press, Tokyo, 1970. MR 42 \# 7951.

Faculty of Engineering, Yokohama National University, Tokiwadai, Hodogaya-Ku, YOKOHAMA, JAPAN 